\title{
Homogenization limit in a graded photonic crystal
}

\author{
Eric Cassan, ${ }^{1, *}$ Jean Dellinger, ${ }^{2}$ Xavier Le Roux, ${ }^{1} \mathrm{~K}$. Van Do, ${ }^{1}$ Frédérique de Fornel, ${ }^{2}$ and Benoît Cluzel ${ }^{2, *}$ \\ ${ }^{1}$ Institut d'Electronique Fondamentale, Université Paris-Sud, Centre National de la Recherche Scientifique, 91405 Orsay, France \\ ${ }^{2}$ Groupe d'Optique de Champ Proche-Laboratoire Interdisciplinaire Carnot de Bourgogne, Unité Mixte de Recherche Centre National de la \\ Recherche Scientifique No. 6303, Université de Bourgogne, Dijon, France
}

(Received 23 July 2013; revised manuscript received 11 September 2013; published 27 September 2013)

\begin{abstract}
The transition between the long-wavelength and the short-wavelength regimes of light propagation in twodimensional graded photonic crystal is investigated using a hyperspectral near-field scanning microscope. The experiments show an invariant quantity of only 1.78 times the lattice period as the criterion for the possible application of homogenization theories. These results are discussed in light of Fourier decomposition of the electromagnetic Bloch waves, and a physical interpretation of the observed transition between the two light propagation regimes is proposed. These results indicate the robustness of the homogenization approaches and suggest that the sharp transition between the two light propagation regimes could be profitably combined in graded optical artificial materials.
\end{abstract}

DOI: 10.1103/PhysRevB.88.125138

PACS number(s): 42.70.Qs, 78.67.Pt, 81.05.Xj

\section{INTRODUCTION}

Photonic crystals $(\mathrm{PhC})$ are artificial optical materials made of a periodic arrangement of dielectric elementary constituents. The optical properties which are often desired for $\mathrm{PhCs}$, such as photonic band gap, ${ }^{1}$ slow light, ${ }^{2}$ or unusual dispersion ${ }^{3,4}$ such as negative and ultrarefraction or self-collimation, are obtained in the short-wavelength regime (SWR) or the diffractive regime. These effects are obtained for a frequency range close to the photonic band gap where the light behavior is governed by the interferences between forward and backward electromagnetic waves. From the classical Bragg relationship, this corresponds to normalized frequencies $\omega=a / \lambda$ equal to $\omega_{B}=(2 n)^{-1}$, where $a$ is the lattice period, $\lambda$ the vacuum wavelength, and $n$ the optical effective index to be considered. For the semiconductors and dielectrics usually employed for planar $\mathrm{PhC}$, the $n$ value ranges from 1.7 to 3 , which corresponds to $\omega_{B}$ values ranging from 0.2 to 0.3 .

Below the photonic band gap $\left(\omega \ll \omega_{B}\right)$, the wavelength becomes much larger than the lattice period, and light then propagates in a homogeneous-like material. This long-wavelength regime (LWR) is described by the theories of homogenization of periodic or composite materials ${ }^{5-8}$ (and references therein). In the most general case, planar PhCs behave as anisotropic biaxial optical media, which give rise to unusual physical properties such as in-plane anisotropy in comparison with natural crystals. ${ }^{9}$ Using a rotationally symmetric lattice cell unit defined both by the Bravais lattice and the geometry of the inclusions, media with long-wavelength in-plane isotropic behaviors can be also defined. This leads to a homogenized index tensor reduced to a single scalar value that can be evaluated in the LWR, i.e., for $\omega \rightarrow 0$, as the square root of the dispersion diagram curve slope. TE (in-plane electric field) and TM (in-plane magnetic field) modes have different slopes, ${ }^{8}$ but only the effective long-range index, $n_{\text {eff }}$, for TM modes has an analytical expression given by ${ }^{8,9}$

$$
n_{\text {eff }}=\sqrt{f \times n_{\text {hole }}^{2}+(1-f) \times n_{\text {diel }}^{2}},
$$

with $f$ as the lattice filling factor, and $n_{\text {hole }}$ and $n_{\text {diel }}$ as the optical index of the hole and the surrounding dielectric media, respectively. Several recent works relying on the formalism of spatial coordinate transforms for the design of artificial optical materials have directly used this homogenized index approach. This regime has attracted an increasing interest with the emergence of metamaterials and their fascinating optical properties. Experimental demonstration of electromagnetic invisibility devices or Luneburg lensing structures, ${ }^{10,11}$ for instance, have been reported.

While LWR and SWR have been separately explored, the gap between them has not received much attention. We provide here a direct experimental observation of an abrupt transition between the two regimes of light propagation in a graded PhC. Thanks to a hyperspectral scanning near-field optical microscope (HSNOM), ${ }^{12,13}$ this transition is observed over a broad spectral range at near-infrared wavelengths for an invariant ratio between the propagating wavelength and the $\mathrm{PhC}$ periodicity. This allows us to define a homogenization limit close to $\lambda / 2.2$, which is a significant much larger value than the $\lambda / 10$ limit usually considered for homogenization theory.

\section{METHODS}

We have considered here a square lattice made of cylindrical air holes etched in a silicon on insulator (SOI) wafer. As shown in Fig. 1(a), the fabricated artificial material is obtained by breaking the lattice symmetry through the introduction of a gradual in-plane modification of the air hole filling factor $(f)$. Lower and higher values of the normalized hole radius range from $r / a=0.20$ to $r / a=0.35$. Light is injected inside the gradual PC with a silicon waveguide, and a HSNOM is used to provide a direct visualization of the light path over a broad spectral range. The near-field measurements are restricted here to propagating wavelengths ranging from $\lambda=1300 \mathrm{~nm}(\omega=$ $0.296)$ to $1550 \mathrm{~nm}(\omega=0.248)$, which correspond to a normalized frequency range where the transition between the SWR and LWR is observed for the TM mode considered throughout this work. A multimedia file showing the continuous variation of the near-field images across the whole spectral range is provided online (see Supplemental Material in Ref. 14). 


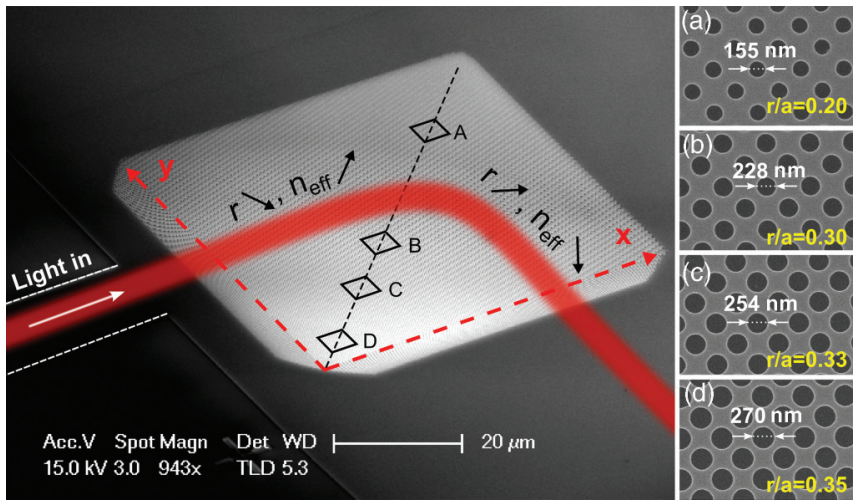

FIG. 1. (Color online) Scanning electron micrographs views of the graded PhC. An overview of the semiconductor sample with insets showing structural details is provided. The periodic lattice is a $45^{\circ}$-rotated square lattice of air holes etched in a 260 -nm-thick silicon on insulator substrate. The lattice period is constant over the structure with $a=385 \mathrm{~nm}$, while the air hole radius is varied along the diagonal from $r / a=0.20$ to $r / a=0.35$.

\section{EXPERIMENTAL RESULTS}

As shown in Fig. 2, from 1300 to 1420 nm, the light path inside the gradual $\mathrm{PhC}$ is characterized by a slightly upward bent path towards the region of smaller $f$ (higher long-range index), whereas the last part of the trajectory is bent downward towards the region of larger $f$ (lower long-range index). In this spectral range, the area of the gradual $\mathrm{PhC}$ where the light is bent downward is simply shifted with the increasing wavelength. The beginning upward bending of the light path can be easily related to conventional mirage effect in the LWR regime, in exact analogy with the atmospheric mirage effect, where the light trajectory is bent towards the regions of high long-range average index $\left(n_{\text {eff }}\right)$. The abrupt downward bending effect is thus the signature of a nonconventional mirage effect, meaning that light propagation has then entered the SWR. Qualitatively, along the first part of the light path, $\lambda / n_{\text {eff }}$ decreases with $f$ to reach a threshold " $\lambda / n$ " value at $\left(x_{\mathrm{th}}, y_{\mathrm{th}}\right)$ where the approximation of homogenization fails. For the longer wavelengths (up to $1450 \mathrm{~nm}$ ), the transition from the LWR to SWR disappears since the " $\lambda / n$ " threshold value is not reached during propagation inside the $\mathrm{PhC}$ area. Thus, the measured light path remains only driven by the conventional mirage effect in the LWR. From $1450 \mathrm{~nm}$, we note a second beam bent by the nonconventional mirage effect, which appears at the entrance of the $\mathrm{PhC}$ area. As reported earlier, ${ }^{13}$ this corresponds to the appearance of a TE mode propagating inside the graded $\mathrm{PhC}$, which is not useful for the scope of this paper. For upper wavelengths,
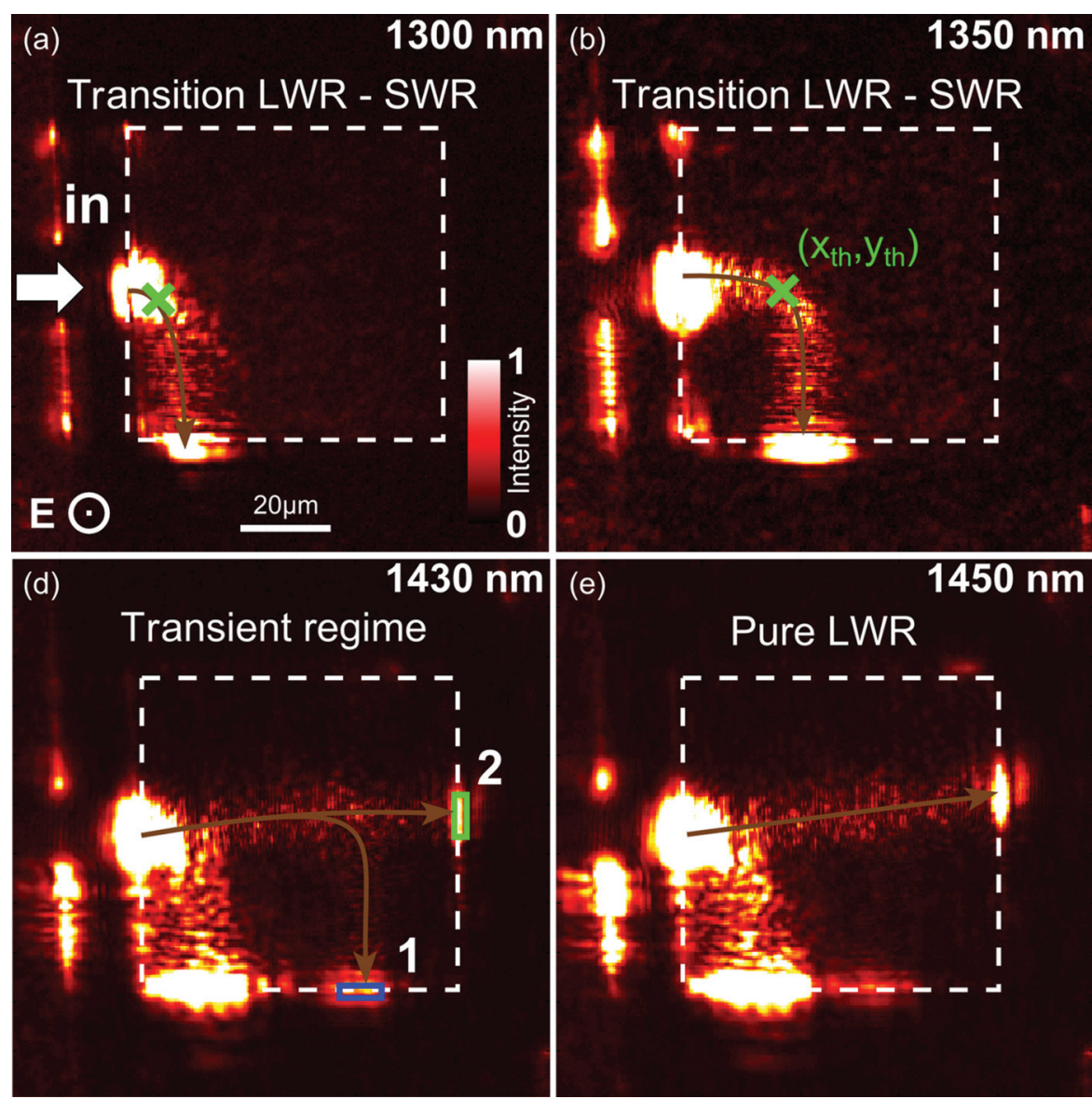
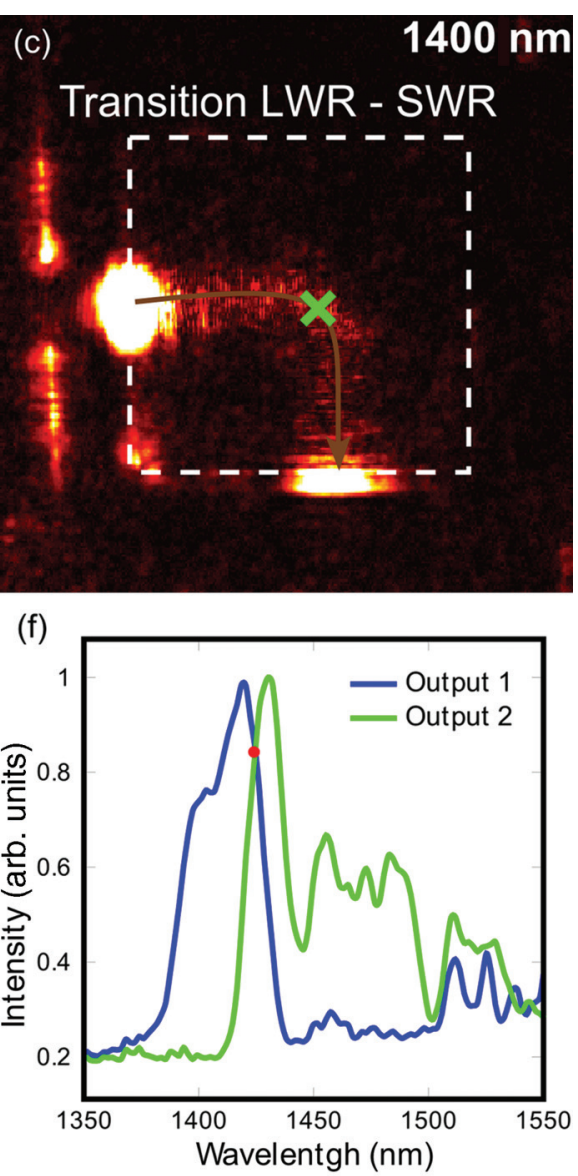

FIG. 2. (Color online) (a)-(e) Near-field observations of the light beam propagation inside the graded PhC. Different wavelengths extracted from the hyperspectral movie available online are shown (see Supplemental Material in Ref. 14). (f) Near-field spectra extracted from the near-field hyper images at positions 1 and 2 shown on the image of Fig. 2(d) at $\lambda=1435 \mathrm{~nm}$. 
both TE and TM modes coexists and are split by the graded PhC. ${ }^{15}$ With the TE mode effective index at the input of the graded $\mathrm{PhC}$ being more up than the $\mathrm{TM}$ one, the propagation regime in TE is similar to the TM one but shifted at upper wavelengths.

More interestingly, a transient regime is observed for wavelengths around $1430 \mathrm{~nm}$ [Fig. 2(d)]. In this range, a splitting of the propagating beam is observed inside the gradual $\mathrm{PhC}$ area. A first part of the beam propagates according to the LWR, while the other one is bent downward and is thus governed by the SWR. As shown on the spectra recorded at the outputs of the two beams, the transient regime is observed on a sharp 10-nm spectral range centered at $1430 \mathrm{~nm}$. This particular regime, which could find some applications for developing integrated beam splitters, is probably related to the finite wave vector distribution of the input beam at the different propagating wavelengths. The careful examination of this transient regime will be detailed hereafter.

The main result of this paper is the observation of the fairly abrupt transition between the LWR and SWR at a threshold location $\left(x_{\mathrm{th}}, y_{\mathrm{th}}\right)$ moving over the spectrum. From the HSNOM images, $\left(x_{\mathrm{th}}, y_{\mathrm{th}}\right)$ can be easily tracked over the spectral range (1300-1440 nm). Then, this measurement enables us to retrieve the corresponding local air hole filling factor $f$. Using Eq. (1), the local threshold long-range index $n_{\text {eff }}$ as a function of the propagating wavelength is then directly obtained. Using $n_{\text {diel }}=2.26$ and $n_{\text {hole }}=1$, corresponding to the average optical effective index of the SOI wafer over the considered spectral range, the experimental ratio $\lambda / n_{\text {eff }}$ is reported in Fig. 3(a). As visible in the figure, this quantity is obviously an invariant that describes the transition between the LWR and the SWR. The average $\lambda / n_{\text {eff }}$ value is measured to be $677.4 \mathrm{~nm}$, i.e., only 1.78 times the lattice period $a$, with a standard deviation as low as $4.4 \mathrm{~nm}$.

\section{DISCUSSION}

For a further interpretation of this invariant quantity, we then converted it into wave-vector modulus assuming that the dispersion relationship $\boldsymbol{k}=n_{\text {eff. }} 2 \pi / \lambda$. holds for each wavelength up to the threshold point. Thus, rescaling the wave vector into a $2 \pi / a$ normalized unit to match with the conventional $\mathrm{PhC}$ notation, the threshold wave vector amplitude $k_{\mathrm{th}}$ is depicted in the right $y$ axis of Fig. 3(a). The obtained average value is 0.573 $[2 \pi / a]$ (with a standard deviation of 0.004 ). Then, the wavevector threshold can be superimposed in the reciprocal space on the first band equifrequency surfaces (EFS) of an infinitely periodical media with $r / a=0.3$. Remarkably, as shown in Fig. 3(b), the experimental LWR/SWR threshold condition corresponds to the region in $\boldsymbol{k}$ space where the change of the EFS curvature occurs. Changing the $r / a$ value from 0.2 to 0.35 only changes the reduced frequency corresponding to the change of curvature but does not modify its $\boldsymbol{k}$ value. As a consequence, the transition between the LWR and the SWR appears as a progressive deformation of the EFS curvature from positive circular shapes centered on $\Gamma$ to negative square shapes centered on the $M$ points close to the Brillouin first zone extrema.

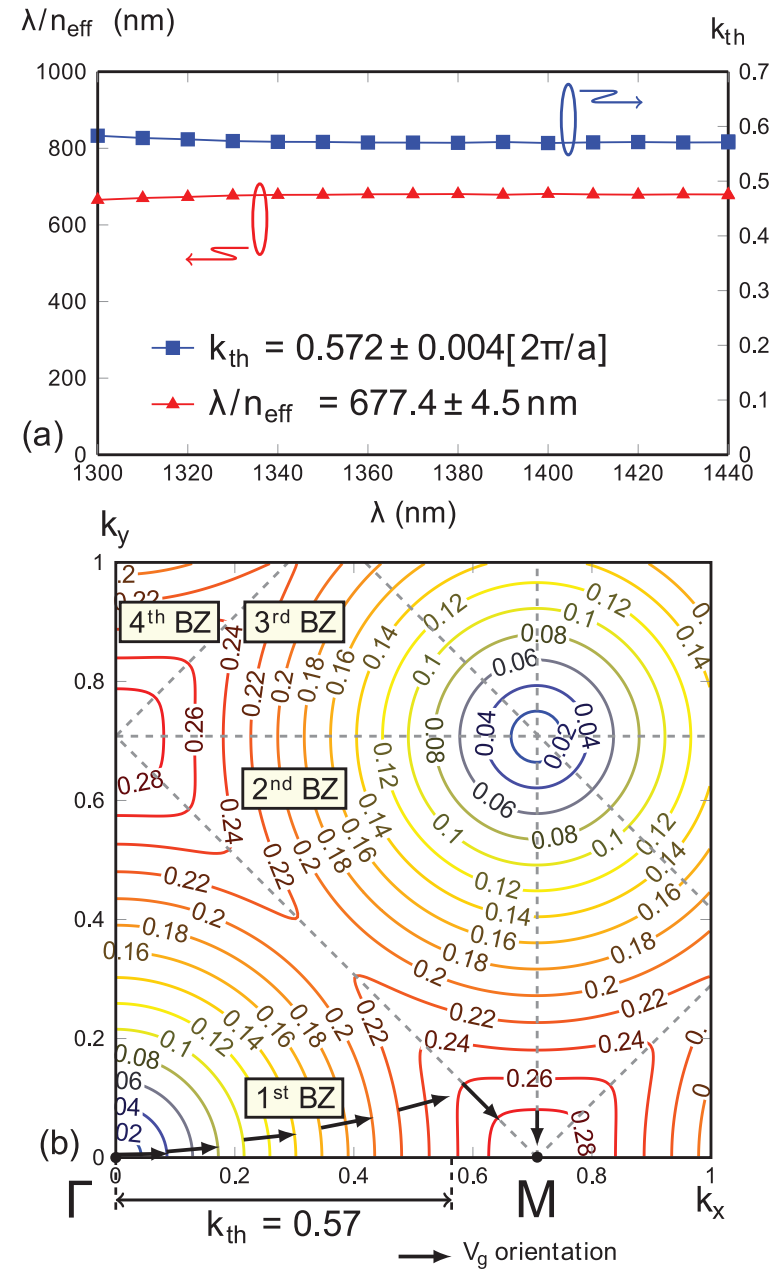

FIG. 3. (Color online) (a) Experimental characterization of the transition between the LWR and SWR of light propagation within the graded $\mathrm{PhC}$ area. The quantity $\lambda / n$ is defined by using a homogenized $\mathrm{PhC}$ refractive index evaluated from the experiments at the threshold position $\left(x_{\mathrm{th}}, y_{\mathrm{th}}\right)$ between the conventional the conventional and unconventional mirage effects typically shown (Fig. 2.). This quantity is recalled as a normalized wave-vector amplitude ( $2 \pi / a$ units) for comparison with the EFS diagrams shown in (b). On the EFS, the arrows highlight the evolution of the group velocity along the wavevector path of the beam inside the graded PhC.

To provide a physical interpretation of this observation, we restart here from the decomposition of the propagating Bloch wave into an infinite series of harmonics: ${ }^{16}$

$$
\boldsymbol{H}(\mathrm{r})=\exp (i \boldsymbol{k} . r) \sum_{\boldsymbol{G}} h_{\boldsymbol{k}}(\boldsymbol{G}) \boldsymbol{H}_{0} \exp (i \boldsymbol{G} \cdot r) .
$$

Here, $\boldsymbol{H}_{\mathbf{0}}$ is the field amplitude, $\boldsymbol{k}$ is the wave vector, $h_{\boldsymbol{k}}(\boldsymbol{G})$ are the dimensionless Fourier coefficients of the Bloch harmonics. The contribution rates $\eta_{\boldsymbol{G}}(\boldsymbol{k})$ to the global Bloch wave energy of each harmonics at point $\boldsymbol{k}$ characterized by a $\boldsymbol{G}$ shift with respect to the $\Gamma$ are obtained $\left(0<\eta_{G}<100 \%\right)$ as $^{15}$

$$
\eta_{\boldsymbol{G}}(\boldsymbol{k})=\left|h_{k}(\boldsymbol{G})\right|^{2} .
$$

As mentioned in Ref. 8, the long wavelength limit of $\mathrm{PhC}$ is satisfied as long as the Fourier expansion of the field 

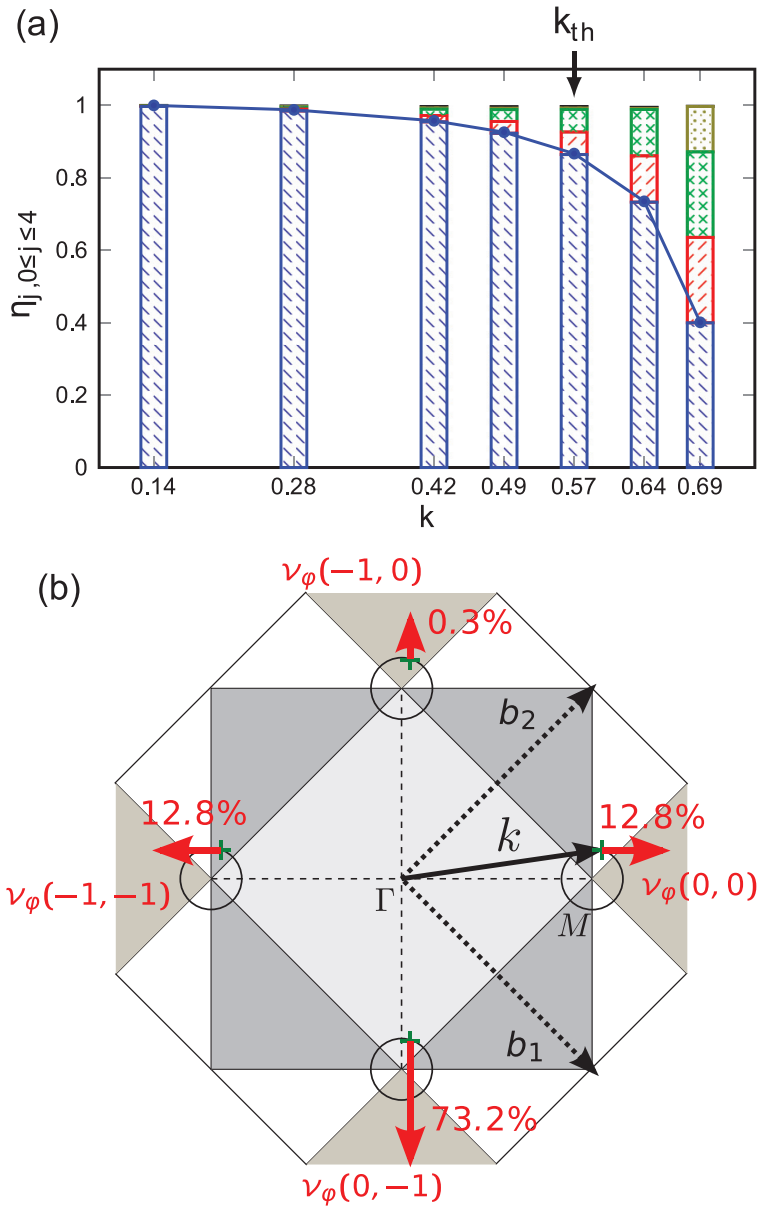

FIG. 4. (Color online) Harmonics decomposition of the Bloch wave inside the graded $\mathrm{PhC}$. (a) Relative contributions rates of the five dominant harmonics $\left(0 \leqslant \eta_{j} \leqslant 100 \%\right.$ with $\left.0 \leqslant j \leqslant 4\right)$ for increasing wave vectors along the $\Gamma-M$ direction. (b) Similar contribution rates of the harmonics for a wave vector slightly lying out of the first Brillouin area onto closed round EFS centered on the $M$ points. The result is plotted for $\omega>0.25$ and $\boldsymbol{k}=0.45 \boldsymbol{b}_{\mathbf{1}}+0.55 \boldsymbol{b}_{\mathbf{2}}$ with $\boldsymbol{b}_{\mathbf{1}}, \boldsymbol{b}_{\mathbf{2}}$ the two lattice primary vectors in reciprocal space. The orientation of the individual phase velocities of the four harmonics are shown by the arrows.

of Eq. (2) is reduced to only the fundamental harmonic: $h_{k}(\boldsymbol{G} \neq 0) \ll h_{k}(\boldsymbol{G}=0)$ when $\omega \rightarrow 0$. To go beyond this qualitative statement, the contribution rates $\eta_{j, 0 \leqslant j \leqslant 4}$ of the first five harmonics along the $\Gamma M$ segment in reciprocal space where the change of the EFS curvature sign occurs have been calculated. For this calculation, the periodical medium eigenvalue/eigenvector Hemholtz equation has been solved for an infinitely periodic square lattice characterized by a given value of $r / a$ using the $\boldsymbol{H}$ formulation of the Helmoltz equation with a truncated plane-wave basis with 289 components. Using the wavelength/wave-vector relationship in band one along the $\Gamma M$ segment, we then calculated the contribution rates $\eta_{j, 0 \leqslant j \leqslant 4}$ as a function of $\boldsymbol{k}$. Figure 4(a) shows the result for $r / a=0.3$ that corresponds to a strong index corrugation in which the material homogenization hypothesis is challenging. As a guide to the eye, the threshold $\boldsymbol{k}$ value at $\boldsymbol{k}_{\text {th }}=0.57$ is pointed by an arrow. For lower $\boldsymbol{k}$ values, the fundamental
Bloch harmonic clearly carries most of the Bloch wave energy. Its contribution is still above $70 \%$ up to $\boldsymbol{k}=0.57$ and then suddenly drops due to the influence of the photonic band edge. This result suggests that the inversion of the EFS curvature can be interpreted as a sharp decrease of the fundamental harmonic contribution $(\boldsymbol{G}=0)$ in the Bloch wave decomposition. However, tracking of the wave vector along the $\Gamma M$ segment does not take into account any possible exit of the current $\boldsymbol{k}$ point out of the first Brillouin zone. Thus, considering the closed shapes of the EFS around the $M$ points for $\omega>0.25$, we now consider an excitation $\boldsymbol{k}$ given by $\boldsymbol{k}=0.45 \boldsymbol{b}_{1}+0.55 \boldsymbol{b}_{2}$, with $\boldsymbol{b}_{\mathbf{1}}, \boldsymbol{b}_{\mathbf{2}}$ the two $\mathrm{PhC}$ primary vectors in reciprocal space [Fig. 4(b)]. Due to the translational shift of any $\boldsymbol{k}$ points by $\mathbf{G}=l_{1} \boldsymbol{b}_{\mathbf{1}}+l_{2} \boldsymbol{b}_{\mathbf{2}}$, with $l_{1}, l_{2}$ two integers, contributions to the Bloch wave energy can arise, as previously, from components located in all Brillouin zones. The four "dominant" harmonics are considered here to include the contributions around the four $M$ points. Figure 4(b) illustrates the result in the $\boldsymbol{k}$ space, showing the contribution rates with the percentage of the four dominant plane waves as well as their phase velocities defined as $\boldsymbol{v}_{\text {phase }}\left(l_{1}, l_{2}\right)=\left(\omega /\left|l_{1} \boldsymbol{b}_{\mathbf{1}}+l_{2} \boldsymbol{b}_{\mathbf{2}}\right|^{2}\right) \cdot\left(l_{1} \boldsymbol{b}_{\mathbf{1}}+l_{2} \boldsymbol{b}_{2}\right)$.

The main contribution to the Bloch wave now corresponds to $h_{\boldsymbol{k}}\left(\boldsymbol{G}=-b_{2}\right)$ and not $h_{\boldsymbol{k}}(\boldsymbol{G}=\mathbf{0})$. As it carries more than $73 \%$ of the Bloch wave energy, its downward phase velocity is dominating the group velocity expansion as a sum of weighted phase velocities: $\boldsymbol{v}_{\text {group }}(\boldsymbol{k})=\sum\left|h_{\boldsymbol{k}}\left(l_{1} \mathbf{b}_{\mathbf{1}}+l_{2} \mathbf{b}_{2}\right)\right|^{2}$. $\boldsymbol{v}_{\text {phase }}\left(l_{1}, l_{2}\right) .{ }^{16}$ This result implies that the transition between the LWR and SWR is not necessarily accompanied by a strong power transfer from the fundamental harmonics to the others providing that the operating wave vector does not approach the $M$ points too closely. In light of these different considerations, an interpretation of the transition between the LWR and SWR in the studied configuration can be obtained by considering the wave-vector path in reciprocal space corresponding to the light beam trajectory depicted in Fig. 2. On the EFS of Fig. 3(b), an illustration of the successive group velocity variation along the light path within the gradual $\mathrm{PhC}$ is plotted. Homogenization of the periodical medium can be considered as a valid approach until the primary wave-vector path crosses the first Brillouin zone boundary. For impinging wave vectors out of the first Brillouin zone, the fundamental harmonic contribution rate remains high but a sudden shift its wave vector by $\boldsymbol{G}=-b_{2}$ occurs to keep it in the first Brillouin zone. Thus, the relationship $h_{\boldsymbol{k}}(\boldsymbol{G}=0) \gg h_{\boldsymbol{k}}(\boldsymbol{G} \neq 0)$ becomes $h_{\boldsymbol{k}}\left(\boldsymbol{G}=-b_{\mathbf{2}}\right) \gg$ $h_{\boldsymbol{k}}\left(\boldsymbol{G} \neq-b_{2}\right)$.

This last analysis finally provides a qualitative understanding of the abrupt regime transition discussed earlier. The input beam inside the graded $\mathrm{PhC}$ exhibits a finite wave-vector distribution related to its initial width. As it propagates inside the graded $\mathrm{PhC}$, this distribution is naturally spread. However, as long as the threshold location $\left(x_{\mathrm{th}}, y_{\mathrm{th}}\right)$ is close enough from the input of the $\mathrm{PhC}$, i.e., for beam propagation lengths in the LWR shorter than the Rayleigh distance, the wave-vector distribution spread at the threshold location remains negligible with respect to the unconventional mirage effect. At $1435 \mathrm{~nm}$, ( $\left.x_{\mathrm{th}}, y_{\mathrm{th}}\right)$ is of the order the Rayleigh distance $(\mathrm{Zr} \sim 60 \mu \mathrm{m})$, and the wave-vector distribution at the threshold now lies as well inside and outside the first Brillouin zone, which gives rise to two distinct Bloch waves with orthogonal group velocities. 


\section{CONCUSIONS}

In conclusion, we have reported the experimental observation of the transition between the LWR and SWR of light propagation in a planar strong index contrast all-dielectric photonic metamaterial using a HSNOM technique making possible to image the involved electromagnetic phenomena in a broad wavelength range. The performed investigations allow evidencing an invariant quantity of the form " $\lambda / n$ " as a criterion above in which the homogenization of the composite material can be applied. The obtained value is as low as 1.78 times the material lattice period and can be attributed to the point at which the wave-vector path in reciprocal space exits the first Brillouin zone limits. The robustness of the medium homogenization reported here is an interesting result for their application as photonic artificial materials in the so-called LWR. Additionally, the reported abruptness of the LWR-SWR transition may also be of interest for the realization of original optical functionalities exploiting graded $\mathrm{PhC}$ operating simultaneously in the two regimes of light propagation.

\section{ACKNOWLEDGMENTS}

The authors acknowledge the regional council of Burgundy for financial funding in the framework of the PHOTCOM project. This project has been performed in cooperation with the Labex ACTION program (Contract No. ANR-11-LABX01-01).
*Corresponding authors: eric.cassan@u-psud.fr and benoit.cluzel@ u-bourgogne.fr

${ }^{1}$ E. Kuramochi, M. Notomi, S. Mitsugi, A. Shinya, T. Tanabe, and T. Watanabe, Appl. Phys. Lett. 88, 041112 (2006).

${ }^{2}$ T. Baba, Nat. Photonics 2, 465 (2008).

${ }^{3}$ T. Matsumoto, S. Fujita, and T. Bava, Opt. Express 13, 10768 (2005).

${ }^{4}$ D. Bernier, X. Le Roux, A. Lupu, D. Morini, L. Vivien, and E. Cassan, Opt. Express 16, 17209 (2008).

${ }^{5}$ S. Datta, C. T. Chan, K. M. Ho, and C. M. Soukoulis, Phys. Rev. B 48, 14936 (1993).

${ }^{6}$ P. Lalanne, Appl. Opt. 35, 5369 (1996).

${ }^{7}$ S. V. Nistor, E. Goovaerts, and D. Schoemaker, Phys. Rev. B 57, 277 (1998).

${ }^{8}$ A. A. Krokhin, P. Halevi, and J. Arriaga, Phys. Rev. B 65, 115208 (2002).
${ }^{9}$ P. Halevi, A. A. Krokhin, and J. Arriaga, Phys. Rev. Lett. 82, 719 (1999).

${ }^{10}$ L. H. Gabrielli, J. Cardenas, C. B. Poitras, and M. Lipson, Nat. Photonics 3, 461 (2009).

${ }^{11}$ A. Di Falco, S. C. Kehr, and U. Leonhardt, Opt. Express 19, 5156 (2011).

${ }^{12}$ J. Dellinger, D. Bernier, B. Cluzel, X. Le Roux, A. Lupu, F. de Fornel, and E. Cassan, Opt. Lett. 36, 1074 (2011).

${ }^{13}$ J. Dellinger, K. V. Do, X. Le Roux, F. de Fornel, E. Cassan, and B. Cluzel, Appl. Phys. Lett. 101, 141108 (2012).

${ }^{14}$ See Supplemental Material at http://link.aps.org/supplemental/ 10.1103/PhysRevB.88.125138 to view the hyperspectral movie.

${ }^{15}$ E. Cassan, K. V. Do, J. Dellinger, X. Le Roux, F. de Fornel, and B. Cluzel, Opt. Lett. 38, 459 (2013).

${ }^{16}$ B. Lombardet, L. A. Dunbar, R. Ferrini, and R. Houdré, J. Opt. Soc. Am. B 22, 1179 (2005). 